\title{
AN EXAMPLE OF A HOMEOMORPHISM OF THE PLANE WHERE THE SET OF $\omega$-LIMIT POINTS IS NOT A CLOSED SET
}

\author{
MICHAEL W. HERO
}

(Communicated by James E. West)

\begin{abstract}
Using a self-map of an interval and its associated inverse limit space we construct an example of a homeomorphism of the plane $h$ such that the set of $\omega$-limit points of $h$ is not a closed set.
\end{abstract}

Let $X$ be a metric space with $f: X \rightarrow X$ continuous. For $n>1$ let $f^{n}$ denote $f$ composed with itself $n$ times. A subset $A$ is said to be a global attractor for $f$ provided that (i) $f(A)=A$; (ii) if $x \in X$ then $d\left(f^{n}(x), A\right) \rightarrow$ 0 as $n \rightarrow \infty$; (iii) if $U$ is open and $A \subseteq U$ then there is an open set $V$ and a positive integer $N$ such that $A \subset V \subset U$, and if $n>N$ then $f^{n}(V) \subset U$. A point $y$ is an $\omega$ (resp. special $\alpha$ ) limit point of a set $Y \subset X$, provided there is a sequence of positive integers $(n(i))_{i=1}^{\infty}$ and a sequence of points $\left(y_{i}\right)_{i=0}^{\infty}$ such that $f^{n(i)}\left(y_{i}\right)=y_{i+1}$ (resp. $\left.f^{n(i)}\left(y_{i}\right)=y_{i-1}\right), \lim _{i \rightarrow \infty} y_{i}=y$, and $y_{0} \in Y$. Denote the set of $\omega$ (resp. special $\alpha$ ) limit points of $Y$ by $\Lambda(f, Y)$ (resp. $S A(f, Y))$.

Proposition 1. There is a homeomorphism $g$ of the plane $\mathbb{R}^{2}$ satisfying the following. (i) $g$ has a periodic point of period $m$ if and only if $m$ is a power of 2 ; (ii) the set $\Lambda\left(g, \mathbb{R}^{2}\right)$ is not a closed set; (iii) $\Lambda\left(g, \mathbb{R}^{2}\right)$ is contained in the global attractor of $g^{-1}$.

Other homeomorphisms of the plane $h$ with $\Lambda\left(h, \mathbb{R}^{2}\right)$ not closed have been constructed using more geometric methods.

Associated with $f$ is the inverse limit space $(X, f)=\left\{\left(x_{0}, x_{1}, x_{2}, \ldots\right)\right\}$ $x_{i} \in X$ and $\left.f\left(x_{i+1}\right)=x_{i}\right\}$, and the induced homeomorphism $\hat{f}:(X, f) \rightarrow$ $(X, f)$ given by $\hat{f}\left(\left(x_{0}, x_{1}, x_{2}, \ldots\right)\right)=\left(f\left(x_{0}\right), x_{0}, x_{1}, x_{2}, \ldots\right)$. If $X$ is compact then $(X, f)$ is compact and the projection $\pi:(X, f) \rightarrow X$ given by $\pi\left(\left(x_{0}, x_{1}, x_{2}, \ldots\right)\right)=x_{0}$ is continuous and closed. For a closed interval $I$ the relationship between the dynamics on $(I, f)$ and $I$ has been studied by Barge and Martin in [1-3]. The spaces $(I, f)$ are examples of what Bing has called "snakelike continua," see [5]. In [4] homeomorphisms of $\mathbb{R}^{2}$ are constructed that realize these spaces as their global attractors. This result is summarized in Proposition 2.

Received by the editors June 3,1991; this paper was presented during the Special Session on Classical Real Analysis at the Joint Mathematics Meetings, Baltimore, MD.

1991 Mathematics Subject Classification. Primary 54H20, 58F08, 58F13. 
Proposition 2. Let $f: I \rightarrow I$ be continuous. Then there is a homeomorphism $h$ of the plane $\mathbb{R}^{2}$ and an embedding $q$ of $(I, f)$ into $\mathbb{R}^{2}$ such that $q((I, f))$ is a global attractor for $h$ and $q \hat{f} q^{-1}=h$ on $q((I, f))$.

For $f: X \rightarrow X$ let $\Lambda^{1}=\Lambda(f, X)$. For every $n>0$, inductively define $\Lambda^{n}=\Lambda\left(f, \Lambda^{n-1}\right)$. The attracting center $\Lambda^{\infty}$. is defined to be the intersection of the sets $\Lambda^{n}$. In [7] it is shown that for self-maps $f$ of the interval $\Lambda^{\infty}=$ $S A(f, I)$.

Proposition 3. Let $f: X \rightarrow X$ be continuous where $X$ is a compact metric space, $h$ a homeomorphism of a metric space $Y$, and $q$ an embedding of $(X, f)$ into $Y$ such that $q((X, f))$ is a global attractor for $h$ and $q \hat{f} q^{-1}=h$ on $q((X, f))$. Then (i) $\pi\left(\Lambda\left(\hat{f}^{-1},(X, f)\right)\right)=S A(f, X)$; (ii) $\pi(\Lambda(\hat{f},(X, f)))=$ $\Lambda(f, X)$; (iii) $\Lambda\left(h^{-1}, Y\right)=q\left(\Lambda\left(\hat{f}^{-1},(X, f)\right)\right)$. Moreover, if $X=I$ then $\pi\left(\Lambda\left(\hat{f}^{-1},(I, f)\right)\right)=\Lambda^{\infty}$.

The proof of this proposition follows more or less from the definitions. Relation (i) in Proposition 3 was pointed out to me by M. Misiurewicz during the Spring Topology Conference in Sacramento, California.

Proof of Proposition 1. Let $f$ be a self-map of the interval such that (a) $f$ has a periodic point of period $m$ if and only if $m$ is a power of 2, and (b) the set of recurrent points $R$ is not a closed set. Such a map is constructed in [6]. In [8] it is shown that for such maps $\Lambda^{\infty}=R$. Let $g$ be the inverse of the homeomorphism $h$ given in Proposition 1. Since $\pi$ is a closed map and $\pi\left(\Lambda\left(\hat{f}^{-1},(I, f)\right)\right)=\Lambda^{\infty}$, we have that $\Lambda\left(\hat{f}^{-1},(I, f)\right)$ is not closed. Since $q$ is an embedding, $\Lambda\left(g, \mathbb{R}^{2}\right)=\Lambda\left(h^{-1}, \mathbb{R}^{2}\right)=q\left(\Lambda\left(\hat{f}^{-1},(I, f)\right)\right)$ is not a closed set. Now (i) follows from property (a) of $f$ and (iii) follows from the fact that $q\left(\Lambda\left(\hat{f}^{-1},(I, f)\right)\right) \subset q((I, f))$.

\section{REFERENCES}

1. M. Barge and J. Martin, Chaos, periodicity, and snakelike continua, Trans. Amer. Math. Soc. 289 (1985), 355-363.

2. _ Dense periodicity on the interval, Proc. Amer. Math. Soc. 94 (1985), 731-736.

3. __ Dense orbits on the interval, Michigan Math. J. 34 (1987), 3-11.

4. _ The construction of global attractors, Proc. Amer. Math. Soc. 110 (1990), 523-525.

5. R. H. Bing, Snakelike continua, Duke Math. J. 18 (1951), 653-663.

6. H. Chu and J.-C. Xiong, A counterexample in dynamical systems of the interval, Proc. Amer. Math. Soc. 97 (1986), 361-366.

7. M. Hero, Special $\alpha$-limit points for maps of the interval, Proc. Amer. Math. Soc. (to appear).

8. J.-C. Xiong, The attracting centre of a continuous self map of the interval, Ergodic Theory Dynamical Systems 8 (1988), 205-213.

Department of Mathematics, Bradley University, Peoria, Illinois 61625

E-mail address: hero1@bradley.bradley.edu 